\title{
A New Densification-Mechanism of Copper Powder Sintered under an Electrical Field
}

\author{
Kunlan Huang ${ }^{\mathrm{a} \& \mathrm{~b}}$, Yi Yang ${ }^{\mathrm{a},}$, Yi Qin ${ }^{\mathrm{b}, *}$, Gang Yang ${ }^{\mathrm{a}}$ and Deqiang Yin ${ }^{\mathrm{a}}$ \\ ${ }^{a}$ School of Manufacturing Science and Engineering, Sichuan University, Chengdu, Sichuan \\ 610065, P.R. China \\ ${ }^{b}$ Centre for Precision-Manufacturing, Dept. of DMEM, University of Strathclyde, James Weir
} Building. 75 Montrose Street. Glasgow G1 1XQ, UK

* Corresponding authors:

E-mail address: yangyi@ @scu.edu.cn; qin.yi@strath.ac.uk

\begin{abstract}
A new sintering mechanism is revealed for copper powder being sintered under the influence from electrical field and force-field during forming of micro-components. The analysis of the microstructure and grain boundary evolution on the sintered samples showed that interface disappearance at contact areas between particles is a continuous process which involves new grain formation and grain refinement during this innovative micro-sintering process. The densification process is therefore different from what is known in a conventional powder sintering process.
\end{abstract}

Keywords: Electric-field activated sintering, Micro-FAST, Copper powder, Grain joining, Grain refinement

Over the last two decades, the development towards miniaturization of products and devices in industries such as electronics, optics, communications, etc. has increased the demand for metallic parts manufactured at micro-scale. During the last 10 years, various micro metal-forming processes have been studied and used to produce a variety of micro-metal-components [1-3] and these efforts were highlighted particularly by the EU large-scale integrated project MASMICRO which researched and produced various manufacturing facilities for micro-manufacturing [4, 5]. 


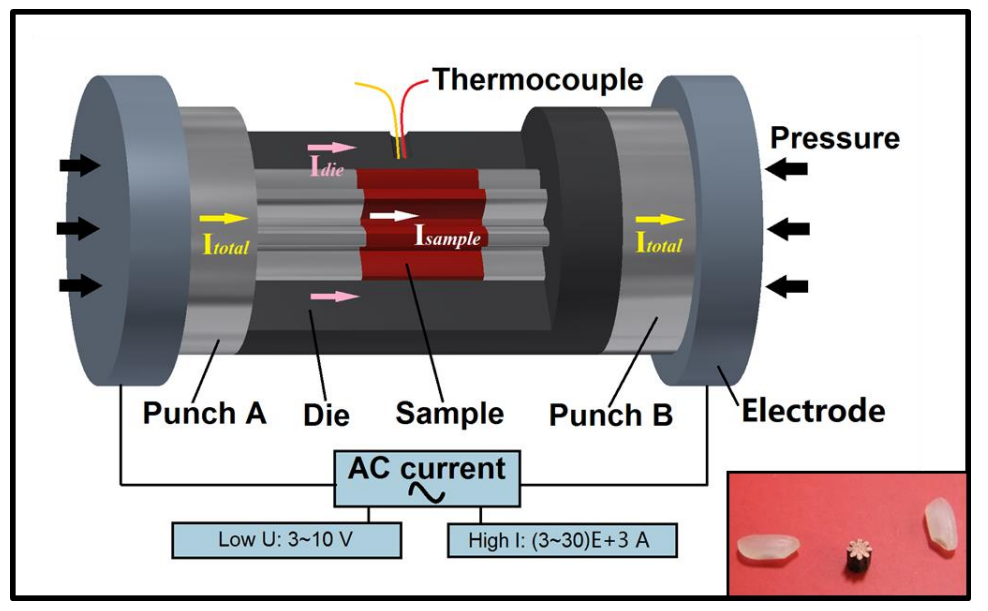

Fig.1 Illustration of the Micro-FAST sintering and forming process

The use of micro-components as well as intensive competition on manufacturing cost led to the requirement for cost-effective production of these components without much compromise on their final quality. To address this issue, a novel micro-forming technology, named Micro-FAST (combining micro-forming and Field-activated sintering technology (FAST)), was proposed recently by the present authors for the forming of micro-components [6-9]: the process is illustrated in Fig.1. Micro-FAST is a method which can be used for the forming of micro-components with a variety of material systems, for instance, copper gears (shown in Fig.1 bottom right corner) have been fabricated successfully at the micro-scale. Over the last few years, pioneering work has been done by several authors and many interesting experimental results have been achieved. Firstly, Micro-FAST is an efficient process, the entire forming process can be accomplished within a few seconds and the relative densities of the formed micro-components are high. Furthermore, for the sintering parameters, the heating rate, sintering temperature and heating cycle have a significant effect on the densification of the metal powders during the sintering process $[6,10]$. Moreover, with continuous high-pressures being applied and fast forming time, Micro-FAST occurs without the coarsening of grains during the densification process [9]. Joule heating is the main heat source during the sintering process and thus it has different densification mechanisms compared to those of conventional processes (including FAST or SPS). Lastly, the densification mechanism of metal powder is related to plastic deformation and the interfacial melting of particles. A three-stage-sintering model has been established to describe the process of the densification of $316 \mathrm{~L}$ 
stainless steel powders when being sintered by Micro-FAST [7]. For the densification process in a traditional powder sintering method, grain growth and neck growth are the critical mechanisms to achieve densification. Grain growth is caused by coarsening which is associated with either surface diffusion or evaporation/condensation. This is why traditional powder sintering would take longer time [11]. The grain coarsening also can be observed in other FSAT methods $[12,13]$. However, the mechanisms of metal powders' sintering neck formation without grain growth for enhanced densification in Micro-FAST remain mostly unexplored.

The purpose of the present work was to study the mechanism of copper powder densification in Micro-FAST based on SEM, TEM and HRTEM analysis. This kind of work has not been done by other researchers. The main objectives were to establish the geometric model of grain evolution between the two particle contact areas during this innovative micro-forming process. The new understanding developed would help to develop new processing strategy for this kind of powder as well as for better quality control during the sintering of micro-components.

Commercially-pure copper powder $(99.7 \%$ purity, $0.05 w t \% \mathrm{~Pb}, 0.05 w t \%$ As, $0.05 w t \% \mathrm{Fe}$, $0.05 w t \% \mathrm{Sb}$ ) with an average particle size of $20 \mu \mathrm{m}$ was used for the experiments. The powders were bought from CNPC POWDER, Shanghai, CHINA. A scanning electron micrograph of the initial powders is shown in Fig.2 (a). The atomized powder also contain a fraction of fine particles with size $<10 \mu \mathrm{m}$.

Table1. The processing parameters corresponding to the samples formed

\begin{tabular}{ccccccc}
\hline $\begin{array}{c}\text { Specimen } \\
\text { designation }\end{array}$ & $\begin{array}{c}\text { Heating } \\
\text { rate }\left({ }^{\circ} \mathrm{C} / \mathrm{s}\right)\end{array}$ & $\begin{array}{c}\text { Pressure on } \\
\text { the punch } \\
(\mathrm{MPa})\end{array}$ & $\begin{array}{c}\text { Sintering } \\
\text { temperature } \\
\left({ }^{\circ} \mathrm{C}\right)\end{array}$ & $\begin{array}{c}\text { Number of the } \\
\text { heating cycle* } \\
\left(\mathrm{T} \sim 300^{\circ} \mathrm{C}\right)\end{array}$ & $\begin{array}{c}\text { Total using } \\
\text { time }(\mathrm{s})\end{array}$ & $\begin{array}{c}\text { Relative } \\
\text { density } \\
(\%)\end{array}$ \\
\hline $1 \#$ & 50 & 75 & 200 & 0 & 48 & 73.46 \\
$2 \#$ & 50 & 75 & 600 & 0 & 76 & 91.27 \\
$3 \#$ & 50 & 75 & 600 & 5 & 148 & 99.23 \\
\hline
\end{tabular}

* A heating cycle is defined as a cycle during which the temperature rises to the maximum value and then drops to about $300{ }^{\circ} \mathrm{C}$

A step-wise experiment has been designed to study the grain evolution due to the fast forming time and the process being carried out in a vacuum $\left(<10^{-4} \mathrm{~Pa}\right)$. In Micro-FAST, the as-received 
powder consisted of agglomerates which were suitable for making up a micro-gear with a pitch diameter of $1.6 \mathrm{~mm}$. After weighing, the loose powders were loaded into a die. Next, the die filled with copper powder was placed into the Gleeble-1500D machine, and then heated rapidly to a particular sintering temperature at a preset heating rate (a high electric current passes through the die set), and at the same time, a preset pressure was applied to the punch. The detailed technological parameters of the experiments are given in Table1.

After sintering, the microstructure of the samples was observed under a scanning electron microscope JSM-5900LV, JEOL (Japan); and the TEM and HRTEM observation was performed using a JEOL JEM-2010F (200 kV TEM) microscope.
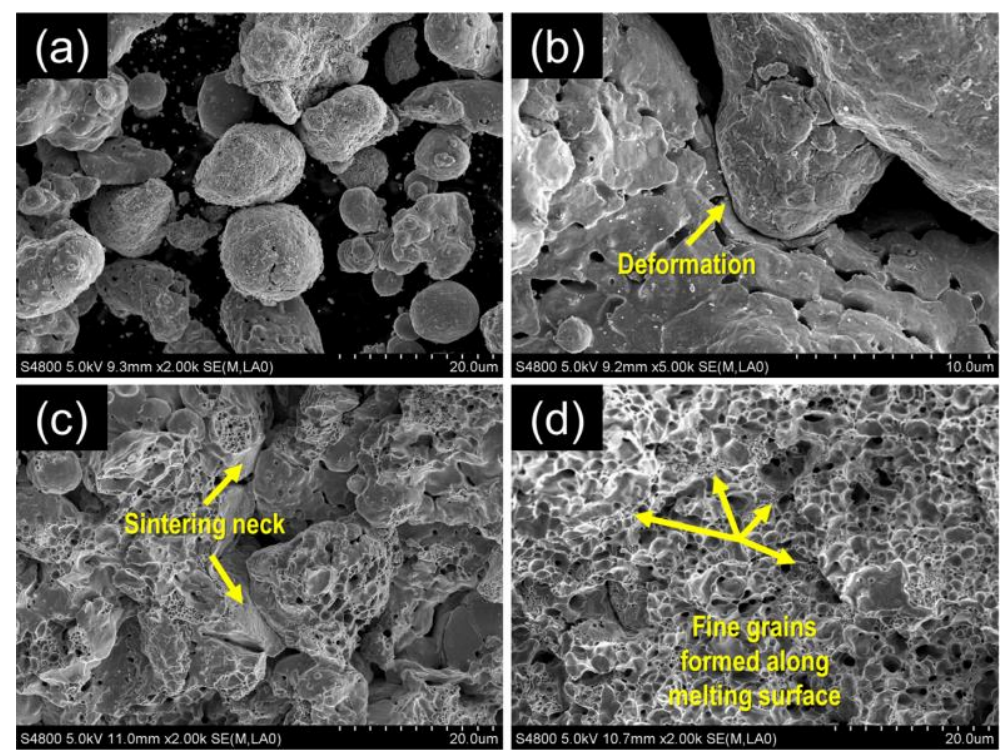

Fig. 2 SEM micrographs of the fracture surface of the samples: (a) the initial copper powders; (b) $1 \#$, sintering at $200{ }^{\circ} \mathrm{C}$; (c) 2\#, sintering at $600{ }^{\circ} \mathrm{C}$; (d) $3 \#$, sintering at $600{ }^{\circ} \mathrm{C}$ with 5 heating cycles.

The SEM micrographs of the fracture surface of the samples sintered at different temperature are shown in Fig.2. Comparing the micrographs in Fig.2, it can be seen that at different sintering levels, the contact state between particles developed from "point contact" to "lineal contact" and then "surface contact". Lu et al [6] have reported that if the temperatures at the contact areas between particles are high, the yield stresses of the material at the contact areas and the viscosity of the compact will decrease. Therefore, the deformation rate of the material at a contact area will be 
greater when the temperature is high, i.e., as the sintering temperature is increased, the deformation rate of the powder compact will increase greatly. Similarly, the distances between particles will decrease under a high pressure, and the contact areas will become larger. The micro-sized pores within the powder compact will become filled with neighboring powder particles due to plastic flow. This is because of the effect of electro-plasticity $[14,15]$, it was found that an electric field of $\sim 1 \mathrm{kV} / \mathrm{cm}$ significantly reduced the plastic flow and fracture stresses of metals at room temperature. It was subsequently determined that the electric field also lowered the brittle-to-ductile transition temperature and reduced the flow stress and increased the elongation at elevated temperatures.

A further important observation is that the formed micro-gear had good mechanical properties. As shown in Figs.2 (c) and (d), the fractograph of the fracture section was of typically dimpled nature, which indicates that the fracture belongs to ductile fracture, and with enlarged dimples and more liquid phase formed [16, 17]. The welded joints between particles observed in Fig.2(c) are clear evidence of the formation of the liquid phase. This figure also shows that provided that a significant amount of heat is generated at the interface of the particles, the melting point of fine powder particles is likely to be achieved at those interfaces that cause the generation of a liquid phase at local areas. Once the liquid phase is formed, it will lead to the melting of the contact surface between particles [18]. At the same time, with the aid of the pressure applied to the compact, the liquid phase flows into the vicinal pores due to viscous flow and capillary force [19], which results in the disappearance of the interface between particles and fast densification of the compact (see Fig.2 (d)).

Moreover, upon investigation of the size of grains inside the particle, it is interesting to note that no coarsening of grains accompanied the process of the densification of the micro-sized compact. However, as shown in Fig.2 (d), when the formed micro-parts nearly obtain full relative density (99.23\%), there was an amount of fine-grains formed along the particles' edge location, especially at the interface melting areas between particles. 


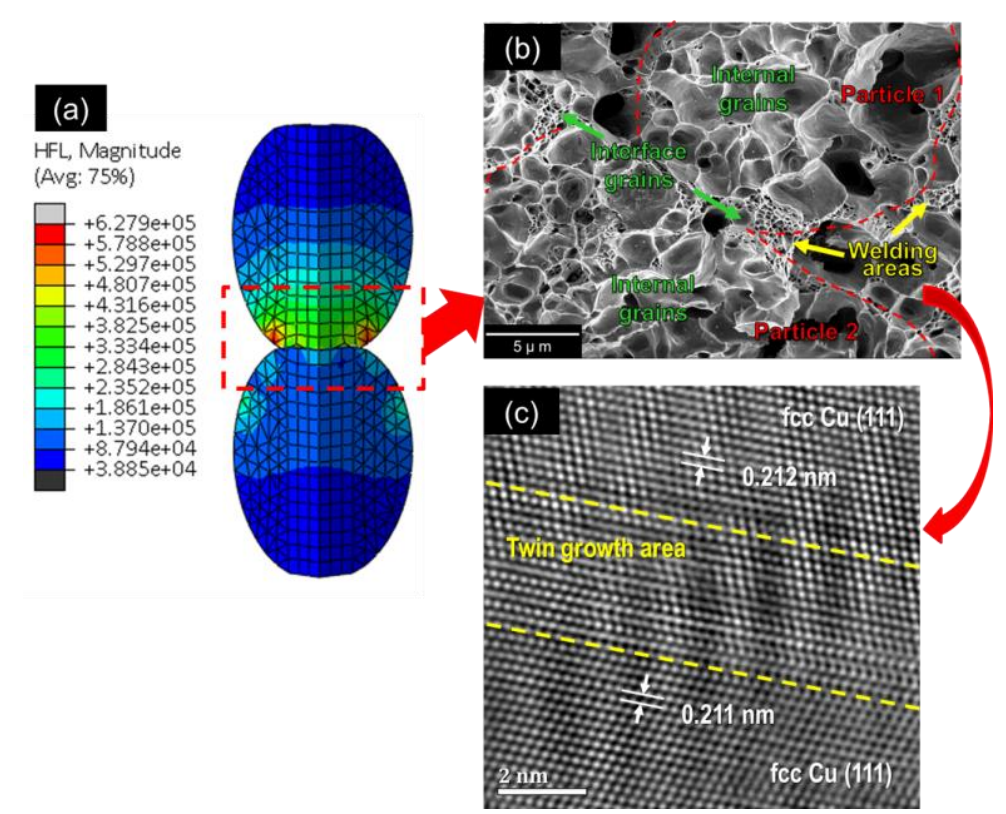

Fig.3. (a) Simulated current density distribution in the two assumed copper-powders; (b) SEM micrograph of the fracture surface; and (c) the HRTEM image of a copper particle in the formed 3\# sample sintered at $600^{\circ} \mathrm{C}$ with 5 heating cycles.

The ABEQUS FE code was used for an electrical-heat analysis. A simulated current density distribution in the two assumed copper powders during the sintering is shown in Fig. 3(a). It can be seen that there was an electric current concentration in the neck area of sintering. This may be a main cause to the particles' deformation to an equilibrium size and the melting of interfaces occurred at this area. This is also evident in the sample shown in Fig. 3(b) where formation of the liquid phase and fine grains between two particles are clearly seen. Furthermore, twin boundary migration (as shown in Fig. 3(c)) is observed and this can become a significant electro-plasticity mode. This investigation strongly suggests that the twin grain growth plays a deterministic role in the structural refinement [20].

We would conclude from these observations that the applied AC field (low voltage: 3 10V; high electric current: $3 \sim 30 \times 10^{3}$ A) leads to welding of the powders/grains in contact within a very short time (a few seconds) in such a fast sintering process (Micro-FAST). Especially, the strong "particles' contact melting" structure is, in some aspects, very similar to certain grain welding structures [21-24]. As soon as these are in contact, e.g. through pressing, in other words, as soon as the separated grains are linked through a neck or necks, a copper powder behaves like an electrical resistor and AC current passes through the powder which induces a Joule heat [21]. 
However, in SPS (spark plasma sintering), as the pulse is very short $(\sim 2 s)$ the generated heat is not enough to really effect sintering, a further dwell sintering time ( $>5$ minutes) appears to be necessary on account of the presence of the neck generated by volume diffusion [25]. The same mechanism also reported in field activated sintering technique (FAST) [26] or electric current assisted/activated sintering (ECAS) [27], promotes densification by the simultaneous action of a high direct current through graphite dies and uniaxial pressure.

Attempting to obtain the strong neck connect between two particles, microstructural studies were focused on interface grains in the "welding areas". As shown in Fig. 3(b), there were a mount of fine-grains formed along the particles 1 and 2 edge location, especially the interface melting areas between the two particles. Grain refinement is one of the most important means of improving its mechanical properties and there are many means to refine the grain, including: increasing the cooling rate in the alloy solidification process and mechanical-physical refinement [28]. In fact, those two methods were involved in the Micro-FAST sintering process. However, according to the fracture surface in Fig.2 and Fig.3, the fracture plane, i.e. hardness reduction, usually happens in the "welding areas", even though the grain size in the areas is remarkably smaller than that of the internal grains. This phenomenon was believed to be that the annealing effect exerts a larger effect than the grains refinements in the mechanical properties of the copper joints [29]. Another important reason is about the residual porosities formed among the "welding areas" (see Fig. 3(c)). During the tensile test, because of stress concentration around the porosities, the micro-crakes usually initiate from these weakest positions, and then propagate to fracture the joints. 


\section{Different sintering level}

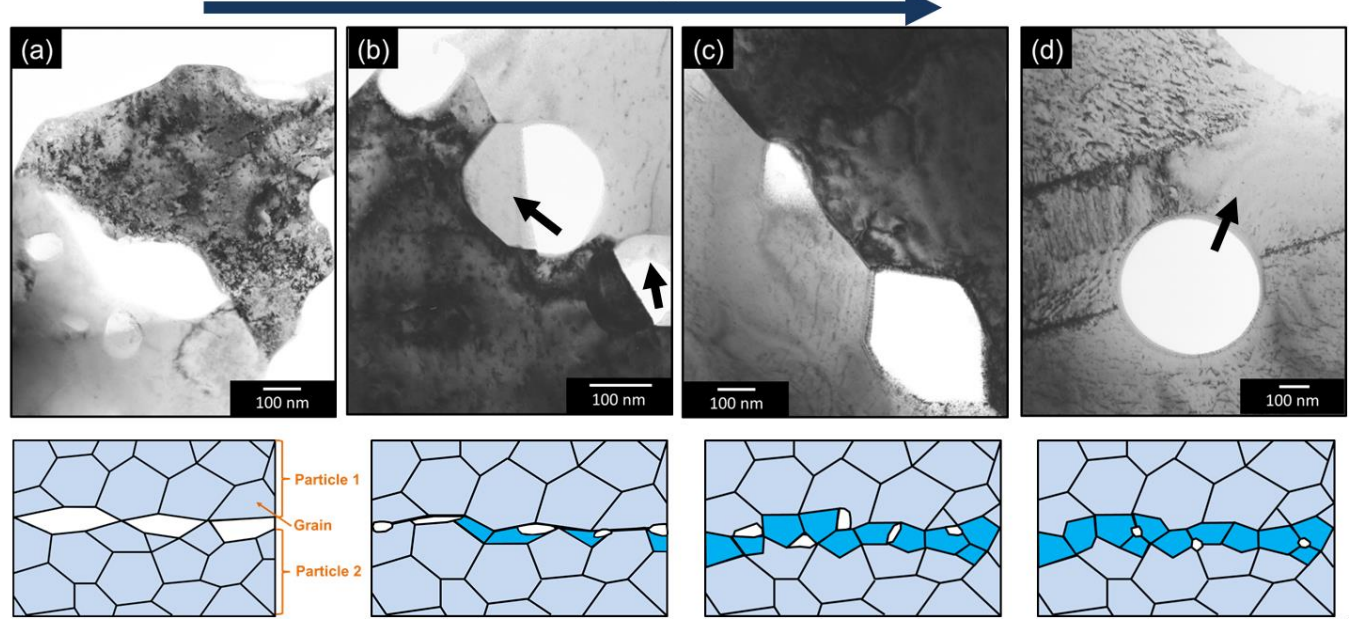

Fig.4 TEM images and model of different stages of grain boundary evolution during Micro-FAST: (a) and (b) $1 \#$, sintering at $200{ }^{\circ} \mathrm{C}$; (c) $2 \#$, sintering at $600{ }^{\circ} \mathrm{C}$; (d) $3 \#$, sintering at $600{ }^{\circ} \mathrm{C}$ with 5 heating cycles.

Fig.4 shows the TEM images of the microstructure of the "welding areas" obtained at different sintering levels, as well as the model of different stages of grain boundary evolution during Micro-FAST.

First, a contact between grains [30] occurs through the mating surfaces (see Fig. 4(a)).

During the second stage (see Fig. 4(b)), some new grains are formed among the grain boundaries [31]. It is more interesting to note that the new grains are formed at such a low temperature (200 $\left.{ }^{\circ} \mathrm{C}\right)$, In fact, the temperature $\left(200{ }^{\circ} \mathrm{C}\right)$ was tested near the sample's surface, and the local temperature is greater due to the local field intensification at inter-particle contact areas. However the total heating time is only a few seconds, which results in the forming of new grains were being incomplete (indicated by the arrow in Figs.4 (b)).

During the third stage (see Fig. 4(c)), diffusion within grain boundaries predominates, thus diminishing pores and ensuring arrangements of grain boundaries. However, the diffusion of grain boundaries was still not complete, because of the short sintering time and high cooling rate $\left(20^{\circ} \mathrm{C} / \mathrm{s}\right)$. Fang et al [32] have reported that the diffusion ability of the copper atoms decreases as the temperature drops and the atoms almost stop moving at the temperature of $600 \mathrm{~K}$. This indicates the system has transformed from the super-cooled liquid state. In fact, the microstructure of the 
metal was changes in several ways during the deformation process at high temperature. First, and most obviously, the grains change their shape and there is a surprisingly large increase in the total grain boundary area. The new grain boundary area (see Fig. 4(b)) has to be created during deformation and this is done by the incorporation of some of the dislocations (see Fig. 3(c) and Fig. 4(a)) that are continuously created during the deformation process. A second obvious feature, recrystallization involved the formation of new strain-free grains in certain parts of the specimen (see Fig. 4(b)) and the subsequent growth of these to consume the deformed or covered microstructure (see Fig. 4(c)). The unqualified term recrystallization always used to mean the primary recrystallization of the deformed microstructure. It is convenient to divide primary recrystallization into two regimes, nucleation which corresponds to the first appearance of new grains in the microstructure and growth during which the new grains replaced deformed material. Although these two events occur consecutively for any particular grain, both nucleation and growth were occurring during the sintering process in Micro-FAST. The kinetics of recrystallization are therefore superficially similar to those of a phase transformation which occurs by nucleation and growth [33].

During the last stage (see Fig. 4(d)), the grain refinement dominates and the sintering process is completed while the nano-sized residual pores still distribute among the grain boundary. From the microstructure characterization shown in Fig.4 (d), twin structure (indicated by the arrow in Fig.4 (d)) is formed after sintering at $600{ }^{\circ} \mathrm{C}$ with 5 heating cycles. Although nano-sized twins can lead to significant increase in flow stress of ultrafine grained pure copper [34], in the present study the width of the twin lamellar formed in the "welding areas" is about several hundred nanometer, which is slightly too small and so that cannot obviously improve the mechanical properties. Furthermore, the dislocation-free area usually forms close to the twin structure and will inevitably decrease the yielding strength of the original hardened materials. Moreover, the presence of porosity in the grain boundary is one of the major concerns during a powder sintering process, even for the mature powder metallurgy method. The high porosity is also one of the most important factors in the reduction of the failure strength of "welding areas". Because of the large freezing temperature range, there is large solidification shrinkage and a high coefficient of linear expansion, after which any blanks in the expanded space becomes pores [35]. 
Nevertheless, the hardness of the "welding areas" can be improved by increasing of the applied load due to the formation of high density of dislocation cells and microstructure refinement [36]. That is to say, the sintering temperature can be designed slightly greater to increase the quality of the liquid phase formed between two particles. At the same time, the value of the pressure applied on the punch also can be increased slightly to gain some more dislocations and help the liquid phase to complete refinement during the cooling process.

In summary, the whole densification process of copper powders in Micro-FAST can be completed within a short time due to the densification process being a continuous process which involves plastic deformation and the interfacial melting of particles. Under the effect of coupled multi-physical-fields, when the particles are deformed to an equilibrium size, the grains at the contact areas between two particles can be welded in a few seconds in this fast sintering process. Moreover the new grains can be formed and the grain refinement will occur during this fast grains-joining process, which lead to the disappearance of the interface between two particles. The densification process is therefore remarkably different from that in a conventional powder sintering process.

\section{Acknowledgements}

This work was primarily funded by the National Nature Science Foundation of China (no.51275322) and the UK Royal Society / China NSFC International Exchanges Programme (no.51311130134) on the collaborative development of a new micro-manufacturing process. The authors are grateful to Dr. Z. Wang (WPI Advanced Institute for Materials Research, Tohoku University, Japan) for his assistance with experiments.

\section{References}

[1] Y. Qin, Micromanufacturing engineering and technology (Access Online via Elsevier, 2010).

[2] W. L. Chan, M. W. Fu, J. Lu and J. G. Liu, Mat Sci Eng A 527 (2010) 6638.

[3] T. Furushima, H. Tsunezaki, K. Manabe and S. Alexsandrov, Int J Mach Tool Manu (2014) 34.

[4] Y. Qin, A. Brockett, Y. Ma, A. Razali, J. Zhao, C. Harrison, W. Pan, X. Dai and D. Loziak, Int J Adv Manuf Tech 47 (2010) 821.

[5] Y. Qin, J Mater Process Tech 177 (2006) 8. 
[6] L. Dong, Y. Yi, Q. Yi and Y. Gang, J Microelectromech S, 22 (2013) 708.

[7] K. Huang, Y. Yang, Y. Qin and G. Yang, Mater Manuf Process 28 (2013) 183.

[8] L. Dong, Y. Yi and Q. Yi, J Mater Res 27 (2012) 2579.

[9] K. Huang, Y. Yang, Y. Qin and G. Yang, Int J Adv Manuf Tech 69 (2013) 2651.

[10] A. Du, Y. Yang, Y. Qin and G. Yang, Mater Manuf Process 28 (2012) 66.

[11] F. F. Lange, J Eur Ceram Soc 28 (2008) 1509.

[12] K. M. Reddy, N. Kumar and B. Basu, Scripta Mater 63 (2010) 585.

[13] J. Narayan, Scripta Mater 68 (2013) 785.

[14] Di Yang and H. Conrad, Scripta Mater 36 (1997) 1431.

[15] H. Conrad, Mat Sci Eng A 287 (2000) 276.

[16] D. Demirskyi, D. Agrawal and A. Ragulya, J Alloy Compd 509 (2011) 1790.

[17] D. Demirskyi, D. Agrawal and A. Ragulya, Mater Lett 64 (2010) 1433.

[18] N. Beri, S. Maheshwari, C. Sharma and A. Kumar, Mater Manuf Process 25 (2010) 1186.

[19] W. D. Kingery, J Appl Phys 30 (1959) 301.

[20] C. S. Hong, N. R. Tao, K. Lu and X. Huang, Scripta Mater 61 (2009) 289.

[21] R. Muccillo, E. N. S. Muccillo and M. Kleitz, J Eur Ceram Soc 32 (2012) 2311.

[22] H. J. Liu, J. C. Hou and H. Guo, Mater Design (2013) 872.

[23] E. Bayraktar, D. Kaplan, L. Devillers and J. P. Chevalier, J Mater Process Tech 189 (2007) 114.

[24] R. W. Fonda, J. F. Bingert and K. J. Colligan, Scripta Mater 51 (2004) 243.

[25] D. M. Hulbert, A. Anders, J. Andersson, E. J. Lavernia and A. K. Mukherjee, Scripta Mater 60 (2009) 835.

[26] D. Demirskyi, H. Borodianska, S. Grasso, Y. Sakka and O. Vasylkiv, Scripta Mater 65 (2011) 683.

[27] A. Cordier, M. Kleitz and M. C. Steil, J Eur Ceram Soc 32 (2012) 1473.

[28] K. S. Kumar and K. Biswas, Surf Coat Tech (2013) 8.

[29] Y. F. Sun and H. Fujii, Mat Sci Eng A 527 (2010) 6879.

[30] J. E. Burke, J Am Ceram Soc 40 (1957) 80.

[31] J. A. Varela, J. A. Cerri, E. R. Leite, E. Longo, M. Shamsuzzoha and R. C. Bradt, Ceram Int 25 (1999) 253.

[32] H. Z. Fang, X. Hui, G. L. Chen and Z. K. Liu, Phys Lett A 372 (2008) 5831.

[33] A. Rollett, F. J. Humphreys, G. S. Rohrer and M. Hatherly, Recrystallization and related annealing phenomena (Elsevier, 2004).

[34] L. Lu, R. Schwaiger, Z. W. Shan, M. Dao, K. Lu and S. Suresh, Acta Mater 53 (2005) 2169.

[35] Y. H. Yin, N. Sun, T. H. North and S. S. Hu, Mater Charact 61 (2010) 1018.

[36] D. H. Nam, S. I. Cha, B. K. Lim, H. M. Park, D. S. Han and S. H. Hong, Carbon 50 (2012) 2417. 